\title{
OTDM-to-WDM Conversion Based on Time-to-Frequency Mapping by Time-Domain Optical Fourier Transformation
}

Palushani, Evarist; Mulvad, Hans Christian Hansen; Galili, Michael; Hu, Hao; Oxenløwe, Leif Katsuo; Clausen, Anders; Jeppesen, Palle

Published in:

I E E E Journal on Selected Topics in Quantum Electronics

Link to article, DOI:

10.1109/JSTQE.2011.2131121

Publication date:

2012

Document Version

Publisher's PDF, also known as Version of record

Link back to DTU Orbit

Citation (APA):

Palushani, E., Mulvad, H. C. H., Galili, M., Hu, H., Oxenløwe, L. K., Clausen, A., \& Jeppesen, P. (2012). OTDMto-WDM Conversion Based on Time-to-Frequency Mapping by Time-Domain Optical Fourier Transformation. I $E$ E E Journal on Selected Topics in Quantum Electronics, 18(2), 681-688.

https://doi.org/10.1109/JSTQE.2011.2131121

\section{General rights}

Copyright and moral rights for the publications made accessible in the public portal are retained by the authors and/or other copyright owners and it is a condition of accessing publications that users recognise and abide by the legal requirements associated with these rights.

- Users may download and print one copy of any publication from the public portal for the purpose of private study or research.

- You may not further distribute the material or use it for any profit-making activity or commercial gain

- You may freely distribute the URL identifying the publication in the public portal 


\title{
OTDM-to-WDM Conversion Based on Time-to-Frequency Mapping by Time-Domain Optical Fourier Transformation
}

\author{
Evarist Palushani, Hans Christian Hansen Mulvad, Michael Galili, Hao Hu, Leif Katsuo Oxenløwe, \\ Anders T. Clausen, and Palle Jeppesen, Member, IEEE
}

\begin{abstract}
This paper reports on the utilization of the timedomain optical Fourier transformation (OFT) technique for serial-to-parallel conversion of optical time division multiplexed (OTDM) data tributaries into dense wavelength division multiplexed (DWDM) channels. The OFT is implemented by using a dispersive medium followed by phase modulation; the latter being achieved by a four-wave mixing process with linearly chirped pump pulses. Both numerical and experimental investigations of the OTDM-to-WDM conversion technique are carried out. Experimental validations are performed on 320- and 640-Gbit/s OTDM data with error-free performance.
\end{abstract}

Index Terms-All-optical demultiplexing, four-wave mixing (FWM), optical time division multiplexing (OTDM), serial-toparallel conversion, spectral compression, wavelength division multiplexing (WDM).

\section{INTRODUCTION}

$\mathbf{T}$ HE OPTICAL time division multiplexing (OTDM) technique is a simple method for high-speed data generation beyond the bandwidth limitation of electronics. Here, different optical pulse streams, called tributaries, originated from the same laser (same central wavelength), are separately encoded by electrically generated data signals. Due to the low duty cycle of their pulses, the tributaries are serially bit interleaved in order to form the OTDM signal. By employing this technique, the generation of serial data signals with a symbol rate up to 1.28 Terabaud has been reported [1]. In the case of wavelength division multiplexing (WDM), each electrical data stream is allocated to an optical channel with its own central wavelength and each generated from a different laser. In contrast to OTDM, WDM channels can overlap in the time domain (parallel to each other) and at the receiver they can be selected by optical filtering. On the other hand, at the receiver side in OTDM systems each of the tributaries is traditionally demultiplexed in separate high-speed switches. Hence, the receiver complexity and power consumption essentially scale with the number of OTDM tributaries. To

Manuscript received November 17, 2010; revised February 11, 2011 and March 12, 2011; accepted March 13, 2011. Date of publication April 29, 2011; date of current version March 2, 2012. This work was supported by the Danish Research Council through NOSFERATU project and FTP postdoctoral grant 274-08-0055.

The authors are with DTU Fotonik, Technical University of Denmark, DK-2800 Kongens Lyngby, Denmark (e-mail: epalu@fotonik.dtu.dk; hchm@ fotonik.dtu.dk; mgal@fotonik.dtu.dk; huhao@fotonik.dtu.dk; lkox@fotonik. dtu.dk; ancl@fotonik.dtu.dk; pjep@fotonik.dtu.dk).

Color versions of one or more of the figures in this paper are available online at http://ieeexplore.ieee.org.

Digital Object Identifier 10.1109/JSTQE.2011.2131121 overcome this problem, a number of schemes for demultiplexing all or several tributaries in a single switch by serial-to-parallel conversion have been proposed [2]-[4]. Among such schemes, a true OTDM-to-WDM conversion is particularly challenging since it requires the converted tributary spectra to conform to the channel spacing of a WDM grid. The reason is that such a conversion necessitates a compression of the broad spectrum of the low duty-cycle tributaries.

In this paper, we describe how to use the time-domain optical Fourier transform (OFT) technique to convert, in a single step, several 10-Gbit/s tributaries of a $64 \times 10 \mathrm{Gbit} / \mathrm{s}$ OTDM signal onto a WDM grid. The OFT is implemented by dispersing the data signal followed by phase modulation based on a four-wave mixing (FWM) process between the data pulses and linearly chirped pump pulses [5]. In this way, the time information of the OTDM signal is mapped onto the power spectrum of the idler generated in the FWM process, hence converting the time-interleaved OTDM tributaries to different wavelengths. At the same time, the spectra of the converted tributaries are compressed as part of the OFT process, enabling their wavelength spacing to conform with a WDM grid. The principle is investigated through numerical simulations followed by experimental demonstrations. In particular, the OTDM-to-WDM conversion is demonstrated for a 640-Gbit/s OTDM signal, from which eight consecutive 10-Gbit/s tributaries are mapped to a $0.8-\mathrm{nm}$ $(100 \mathrm{GHz})$ channel spaced grid. A full system evaluation with bit error rate (BER) characterization is performed. Investigation of different spectral compression rates is carried out, and numerical simulations show very good agreement with the experimental results.

\section{BASIC PRINCIPLE}

The time-to-frequency conversion process is well described by the similarities between a temporal optical system manipulating pulses of light and a spatial optical system manipulating beams of light. An equivalent temporal optical system is found for a spatial optical one by exchanging spatial variables with temporal variables and spatial frequencies with spectral ones [6]. The effects of diffraction and spatial lenses on a beam of light are equivalent to the effects of dispersion and quadratic phase modulators on a pulse of light [7]. In spatial optics, if the object is placed at the front focal plane of a lens, then the field distribution at this point and at the output plane of such lens are related by a Fourier transform [8]. But, also the output field distribution and its spatial spectral frequency distribution are related by a Fourier transform as well. Therefore, the output 


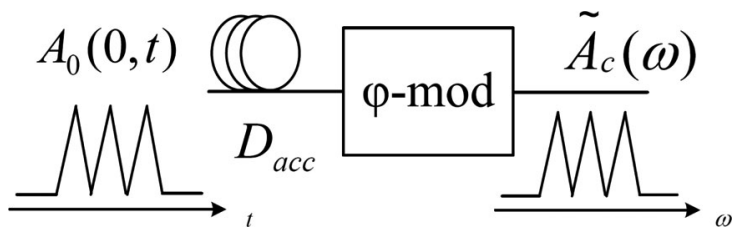

Fig. 1. Schematic of the basic principle of the time-domain OFT technique for time-to-frequency conversion.

spectrum and input field are related by two successive Fourier transforms, implying that measuring the output power spectrum gives the spatial shape of the input object.

The same idea in temporal optics (time-to-frequency conversion) would require the waveform to be converted to travel through a dispersive material followed by a quadratic phase modulation [9] as shown in Fig. 1. In the following section, we will derive the equations describing this time-to-frequency mapping process or time-domain OFT technique.

The transfer function $H_{\beta_{2}}(z, \omega)$ describing propagation through a dispersive medium with second-order chromatic dispersion $\beta_{2}$ and length $z$ is given by

$$
H_{\beta_{2}}(z, \omega)=\exp \left(\frac{i \beta_{2} z \omega^{2}}{2}\right) .
$$

A quadratic phase modulation, imposing a linear chirp $C$, is described in the frequency domain by [10]

$$
H_{l}(\omega)=\sqrt{\frac{2 \pi i}{C}} \exp \left(\frac{-i \omega^{2}}{2 C}\right) .
$$

Let $A_{0}(0, t)$ be the electric field envelope of a waveform at the input of the OFT system, propagated through a dispersive medium of length $L$ with accumulated dispersion $D_{\text {acc }}=\beta_{2} L$. The Fourier transform of the waveform at the output of the dispersive medium is

$$
\tilde{A}_{0}(L, \omega)=\tilde{A}_{0}(0, \omega) H_{\beta_{2}}(L, \omega)
$$

where $\tilde{A}_{0}(0, \omega)$ is the Fourier transform at the input. The dispersed waveform is then quadratically phase modulated $(\varphi$-mod) and its Fourier transform becomes

$$
\begin{aligned}
\tilde{A}_{c}(\omega) & =\frac{1}{2 \pi} \tilde{A}_{0}(L, \omega) * H_{l}(\omega) \\
& =\frac{1}{2 \pi} \int_{-\infty}^{+\infty} \tilde{A}_{0}\left(L, \omega^{\prime}\right) H_{l}\left(\omega-\omega^{\prime}\right) d \omega^{\prime} \\
& =\sqrt{\frac{i}{2 \pi C}} \int_{-\infty}^{+\infty} \tilde{A}_{0}\left(L, \omega^{\prime}\right) \exp \left(-\frac{i\left(\omega-\omega^{\prime}\right)^{2}}{2 C}\right) d \omega^{\prime} \\
& =b \int_{-\infty}^{+\infty} \tilde{A}_{0}\left(0, \omega^{\prime}\right) \exp \left(i \frac{\left(\beta_{2} L C-1\right) \omega^{\prime 2}+2 \omega \omega^{\prime}}{2 C}\right) d \omega^{\prime}
\end{aligned}
$$

where

$$
b=\sqrt{\frac{i}{2 \pi C}} \exp \left(-\frac{i \omega^{2}}{2 C}\right) .
$$

If the condition $C=1 / D_{\text {acc }}$ is fulfilled, then (4) can be written as

$$
\tilde{A}_{c}(\omega)=b \int_{-\infty}^{+\infty} \tilde{A}_{0}\left(0, \omega^{\prime}\right) \exp \left(i \frac{\omega \omega^{\prime}}{C}\right) d \omega^{\prime} .
$$

By using the transformation $t=\omega / C$, the output from the OFT system (6) can be written as

$$
\tilde{A}_{c}(\omega)=2 \pi b A_{0}(0, t)_{t=\omega / C}
$$

and the power spectrum at the output of the OFT (6) becomes

$$
\left|\tilde{A}_{c}(\omega)\right|^{2}=\frac{2 \pi}{|C|}\left|A_{0}(0, t)_{t=\omega D_{\mathrm{acc}}}\right|^{2} .
$$

Equation (8) shows that the power spectrum of the output waveform is proportional to the intensity of the input signal. The dispersion $D_{\text {acc }}$, or equivalently $C$, determines the scaling factor between the time and frequency domains expressed as $t=\omega / C$ and is used for deriving (7). Higher $D_{\text {acc }}$ leads to higher spectral compression. For example, consider an OTDM data signal as initial waveform, consisting of tributary pulses spaced by a time interval $\Delta t$. As a result of the OFT, these tributaries will be mapped to different wavelength allocations spaced by $\Delta \omega=\Delta t C$. The chirp factor $C$, or equivalently $D_{\text {acc }}$, can be used to control the size of the wavelength spacing, e.g., to match a particular WDM grid.

In order to achieve high chirp rates $C$, the quadratic phase modulation can be implemented via parametric processes, such as FWM [5]. In this case, the dispersed waveform to be transformed acts as signal $E_{s}(t)$ and the phase modulation is applied using linearly chirped pump $E_{p}(t)$ pulses, generated by propagation of transform limited pulses in a dispersive medium. In the FWM process, the signal is converted to an idler $E_{i}(t)$, which combines the phases of both pump and signal $\left(E_{i}(t) \propto E_{p}^{2}(t) E_{s}^{*}(t)\right)$. In our case, the signal is $A_{0}(L, t)$, and the idler is $A_{c}(t)$. As a result of the FWM process, the time information contained in the signal is mapped onto the power spectrum of the generated idler.

To achieve an OTDM-to-WDM conversion by the aforementioned technique, the pump pulses must run at the repetition rate of the OTDM tributaries in order to map each tributary bit to the same wavelength. After the time-domain OFT, the idler spectrum will resemble a wavelength grid where each channel is an OTDM converted tributary. This grid can be made to comply with recommendation ITU-T G.694.1 [11], which determines the optical frequencies to be used to identify dense wavelength division multiplexing (DWDM) channels for different frequency grids in the range of $25-200 \mathrm{GHz}$.

\section{NUMERICAL ANALYSIS}

In this section, we numerically investigate [12] the OTDMto-WDM conversion based on the OFT technique. Numerical simulations will examine the idler's shape and channel spacing based on shape and chirp of pump pulses, and signal dispersion. This analysis will help understand the limitations of the OFT technique for OTDM-to-WDM conversion, and clarify which are the system requirements in order for the resulting idler to comply with a certain DWDM grid [11]. 
The OTDM signal is a $640-\mathrm{GHz}$ unmodulated pulse train (64 tributaries at $10 \mathrm{GHz}$ ) having Gaussian-shaped pulses with $0.6 \mathrm{ps}^{1}$ full-width at half maximum (FWHM). Pump and tributaries have the same repetition rate of $10 \mathrm{GHz}$. The highly nonlinear fiber (HNLF) used for the FWM process is $100 \mathrm{~m}$ long and has nonlinear coefficient $\gamma=10(\mathrm{~W} \cdot \mathrm{km})^{-1}$. The FWM conversion efficiency is assumed to be uniform across the entire bandwidth of the signals (data, pump, and idler) involved in the FWM process, and the phase mismatch is assumed to be zero. Under these conditions, the utilization of linearly chirped flattop pump pulses would equalize the WDM channels resulting from OTDM-to-WDM conversion. Such pulses can be obtained by dispersing narrow sinc-like pulses having a broad flat-top spectrum. In this case, the dispersion process maps the spectral profile into the time domain [13] (frequency-to-time mapping). The medium used to disperse the signal, and to linearly chirp the pump is dispersion compensating fiber (DCF) with $\beta_{2}=$ $148 \mathrm{ps}^{2} / \mathrm{km}$.

Fig. 2(a) shows the data, pump, and idler spectra at the output of the HNLF when the pump spectrum is a sixth-order super-Gaussian with $10 \mathrm{~nm}$ FWHM. The probe is dispersed in $L=32 \mathrm{~m}$ DCF in order to achieve at the idler a channel frequency spacing $\Delta f=T /\left(D_{\text {acc }} 2 \pi\right)$ of $50 \mathrm{GHz}$ ( $T=1.56$ ps: OTDM tributary spacing). This corresponds to a linear chirp $C=0.21 \mathrm{ps}^{-2}$, which can be achieved by dispersing the pump in $64 \mathrm{~m}$ DCF. Because of the flat intensity profile of the pump pulse, which broadens up to 74 ps FWHM, it is possible to have half of the tributaries (32 channels) mapped in the frequency domain, well equalized, and having $<1.5 \mathrm{~dB}$ difference. With two of such OTDM-to-WDM converters, it is possible to extract all 64 OTDM channels.

The simultaneous mapping and well equalization of all the WDM channels is hardly possible, as this would require that the pump pulse should have a proper linear chirp, be perfectly square, have the width of the tributary bit slot, and should not interfere with the neighboring pump pulses. This means that the OTDM channels further from the pump center will not be converted with the same intensity as the central ones. Higher compression ratios require higher pump and signal dispersion. This can be seen in Fig. 2(b), where pump and signal are dispersed in 128 and $64 \mathrm{~m} \mathrm{DCF}$ in order to achieve a 25-GHz grid. As the required dispersion is twice as much compared to Fig. 2(a), the spectral FWHM of the pump should be almost half of the previous case $(5.2 \mathrm{~nm})$, in order for the pump pulses to be flat, not interfere with each other, and be 75 ps wide. A spectrally broader pump cannot be used for such a high compression ratio as the broadened pump pulses would overlap with each other, resulting in distorted time-to-frequency mapping. Fig. 2(b) also shows an open eye diagram of one of the converted tributaries. In order to get the eye diagram, the OTDM tributaries were ON-OFF keying (OOK) modulated and the desired channel was selected with a $0.16-\mathrm{nm} 3-\mathrm{dB}$ bandwidth optical bandpass filter (OBPF).

The utilization of Gaussian pump pulses would result in unequal power levels for the WDM channels at the idler. This is related to different pump intensities that various OTDM chan-

${ }^{1} 40 \%$ duty cycle.

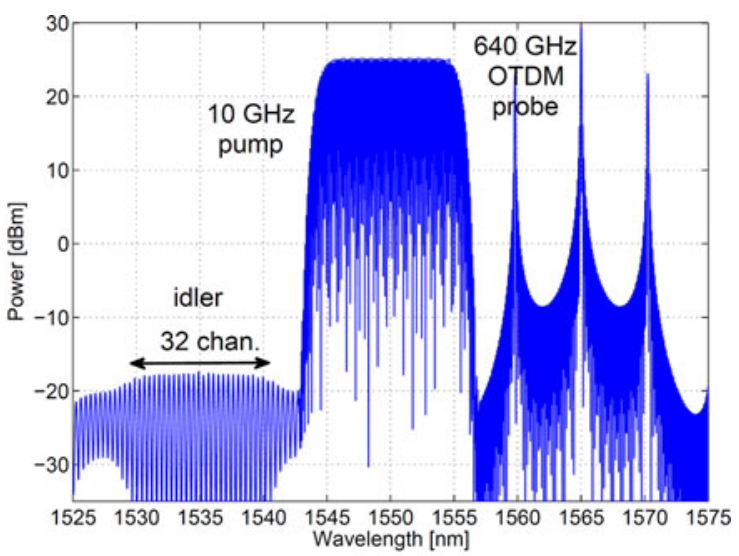

(a)

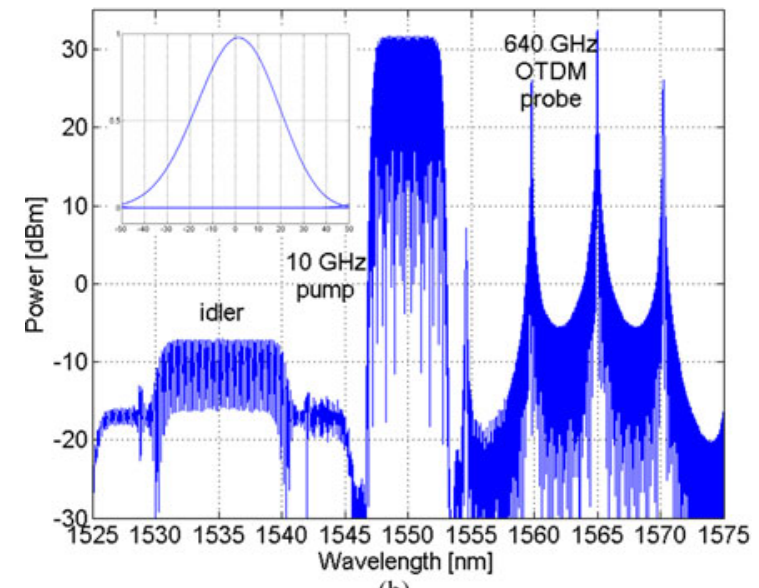

(b)

Fig. 2. Simulated spectra at the output of the HNLF when flat-top, linearly chirped pump pulses are used for OTDM-to-WDM conversion: (a) 50-GHz grid and (b) 25-GHz grid with eye diagram (inset) of one of the converted tributaries.

nels experience. This can be seen in Fig. 3, where pump (10 GHz, 0.5 -ps FWHM Gaussian pulses) and signal are dispersed in, respectively, 32 and $16 \mathrm{~m} \mathrm{DCF}$, resulting in 0.8-nm $(100 \mathrm{GHz})$ channel spacing. Fig. 4 shows the idler for both $100-\mathrm{GHz}$ and $50-\mathrm{GHz}(0.4 \mathrm{~nm})$ spacing. In the latter case, pump and signal are dispersed, respectively, in 64 and $32 \mathrm{~m}$ DCF. Because of the pump's spectral extension and in order to avoid overlap between pump and idler spectra, the wavelength separation between pump and signal is made sufficiently larger compared to the previous cases.

The deviation of both signal and pump chirp from the optimum value for a certain spectral compression would result in spectral distortions of the idler and consequently as well for the selected WDM channels. Fig. 5 shows the eye diagrams for one of the OTDM tributaries when the dispersion of data and pump is separately increased by $10 \%$ compared to the relative optimum case for $100-\mathrm{GHz}$ grid shown in Fig. 4. Since the condition $C=1 / D_{\text {acc }}$ is not fulfilled, the pulses will have a residual phase component which induces some temporal shifts. The chosen WDM channel corresponds to the OTDM tributary overlapping ${ }^{2}$ in the time domain with the center of the

\footnotetext{
${ }^{2}$ This OTDM tributary is always mapped to the same WDM channel, independently on the spectral compression factor.
} 


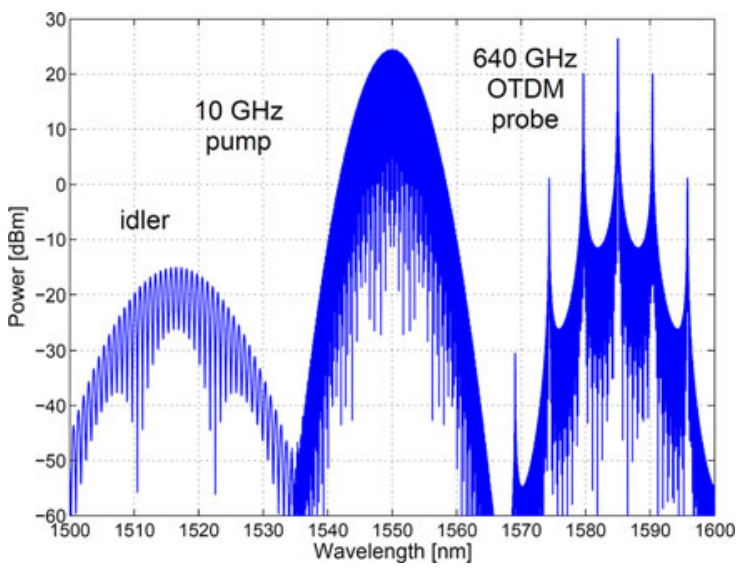

Fig. 3. Simulated spectra at the output of the HNLF when Gaussian, linearly chirped pump pulses are used for OTDM-to-WDM conversion, resulting in a $100-\mathrm{GHz}$ grid.

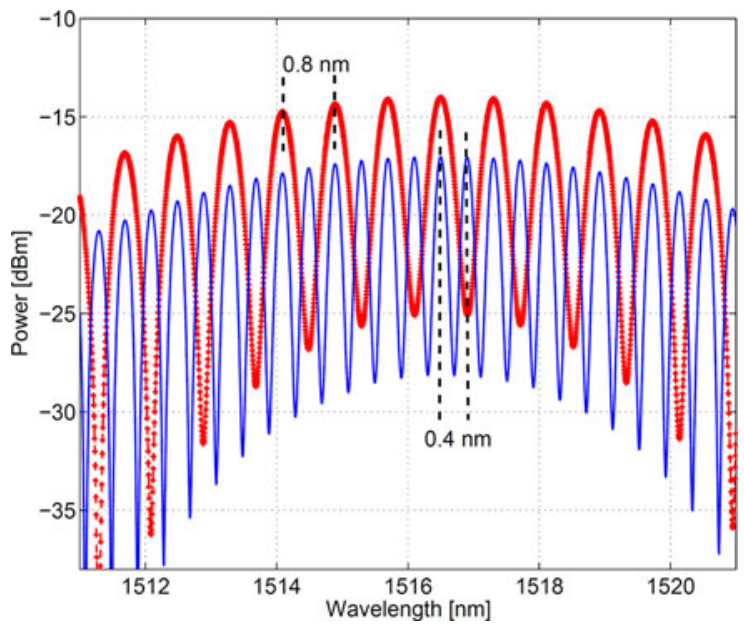

Fig. 4. Simulated spectra at the output of the HNLF when Gaussian pump pulses are used for OTDM-to-WDM conversion, in the case of a $100-\mathrm{GHz}$ $(0.8 \mathrm{~nm})$ and a $50-\mathrm{GHz}(0.4 \mathrm{~nm})$ grid.

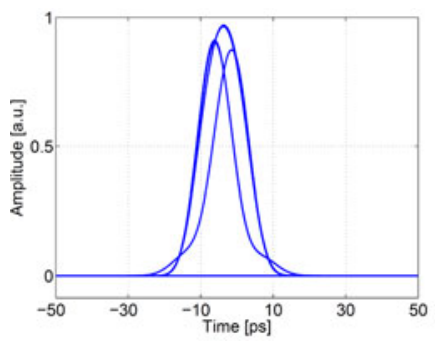

(a)

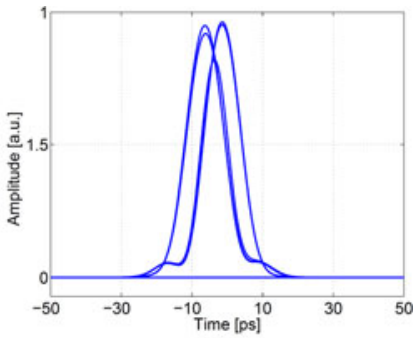

(b)
Fig. 5. Distorted eye diagram when the dispersion of data (a) and pump (b) is independently increased by $10 \%$ compared to the relative optimum case for $100-\mathrm{GHz}$ frequency spacing of Fig. 4.

pump pulse and it was selected with a $0.3-\mathrm{nm} 3-\mathrm{dB}$ bandwidth OBPF. As can be seen, the deviation from the optimum of one of the signals' dispersion is enough to deteriorate the system's performance.

In some cases, the effect of higher order dispersion terms cannot be neglected and should be taken into consideration when studying pulse evolution. The higher order terms of the Taylor

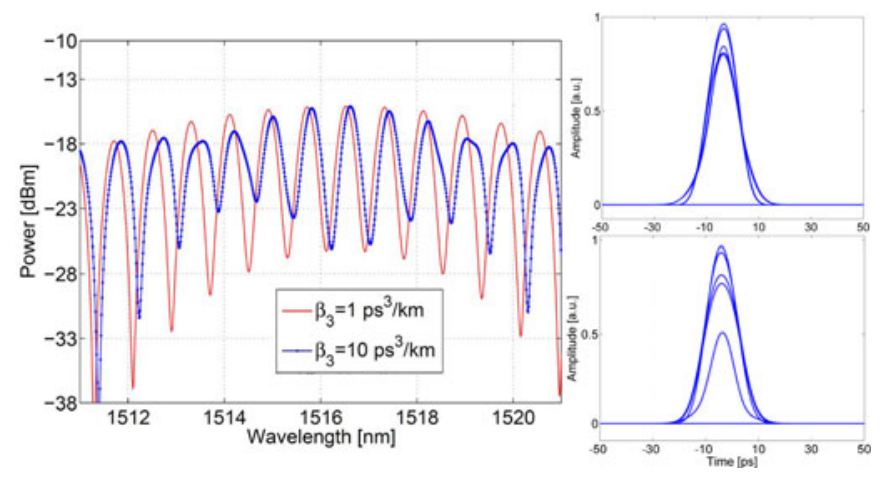

Fig. 6. Simulated idler spectrum showing the effect of $\beta_{3}=1 \mathrm{ps}^{3} / \mathrm{km}$ and $10 \mathrm{ps}^{3} / \mathrm{km}$. To be compared with $\beta_{3}=0$ in Fig. 4. Eye diagrams for $\beta_{3}=$ $10 \mathrm{ps}^{3} / \mathrm{km}$ only for (top) pump dispersion and (bottom) data dispersion.

expansion of the propagation constant $\beta$ come mainly into play for ultrashort pulses, having a broad spectral content, and when the central wavelength is near to the zero dispersion wavelength of the fiber $\lambda_{0}$. For wide spectra, the effect of third-order dispersion (TOD) has to be taken into consideration. TOD is expressed by the term $\beta_{3} \equiv d \beta_{2} / d \omega$, which stems from the variation of the GVD $\left(\beta_{2}\right)$ with frequency, and represents an aberration factor, introducing distortions in the time-to-frequency mapping. Fig. 6 shows the effect of TOD for the $100-\mathrm{GHz}$ spacing scenario of Fig. 4 but with $\beta_{3}=1 \mathrm{ps}^{3} / \mathrm{km}$ and $\beta_{3}=10 \mathrm{ps}^{3} / \mathrm{km}$ for both signal and data fibers. $\beta_{3}=1 \mathrm{ps}^{3} / \mathrm{km}$ is a typical value for commercially available DCF [14] and it does not introduce any distortion in the time-to-frequency mapping. Fig. 6 also shows the eye diagrams when $\beta_{3}=10 \mathrm{ps}^{3} / \mathrm{km}$ only for the pump (top) or only for the signal (bottom). Considerable abberations in the idler spectrum become visible when $\beta_{3}$ reaches or passes this value, for either one of the dispersion media.

Time-to-frequency mapping can also be achieved for higher bit rates, such as 1.28 Tbits/s [1] (see Fig. 7). In this case, the pulses for both probe and pump (at $10 \mathrm{GHz}$ ) are Gaussian with 0.3 -ps FWHM. Because of the closer tributary position in the time domain, half of the dispersion fiber is required in order to achieve the same $100-\mathrm{GHz}$ grid seen in Fig. 4. The resulting spectral compression can be seen in Fig. 7. Because of the closer spacing of the original OTDM tributaries, the extinction ratio between the WDM channels is not as good as in the $640-\mathrm{GHz}$ case (see Fig. 7), generated from the terabaud signal when every second pulse is suppressed. Despite this fact, it is possible to get an open eye (see the inset in Fig. 7) by filtering with a narrower filter (0.2-nm 3-dB bandwidth).

\section{EXPERIMENTAL RESULTS}

\section{A. Experimental Setup}

Fig. 8 shows the schematic of the experimental setup used to perform the OTDM-to-WDM conversion. The same setup is used to test the principle on both 320- and 640-Gbit/s OTDM systems, except for some small details concerning optical bandpass filtering and power levels. The pulse source is an erbium glass oscillating pulse-generating laser (ERGO-PGL) emitting 1.5-ps FWHM pulses with $10-\mathrm{GHz}$ repetition rate and centered at $1542 \mathrm{~nm}$. The ERGO-PGL output is amplified and its 


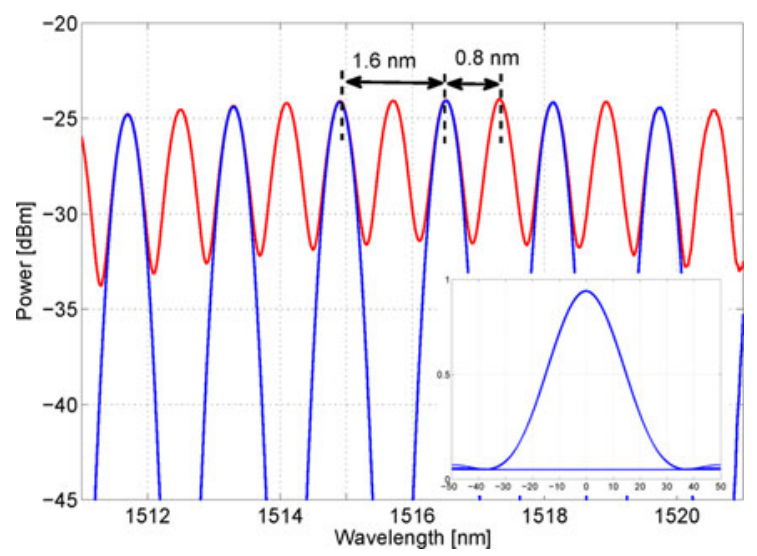

Fig. 7. Simulated idler spectrum: $100-\mathrm{GHz}(0.8 \mathrm{~nm})$ spacing and $200-\mathrm{GHz}$ $(1.6 \mathrm{~nm})$ spacing for, respectively, $1.28-\mathrm{THz}$ and 640-GHz OTDM-to-WDM converters when pump and data are dispersed in 16- and 8-m fiber with $\beta_{2}=$ $148 \mathrm{ps}^{2} / \mathrm{km}$. Inset: eye diagram of one of the $10-\mathrm{Gbit} / \mathrm{s}$ converted tributaries of the 1.28-Tbit/s OTDM signal.

spectrum is broadened through self-phase modulation, generating a supercontinuum in a 400-m dispersion-flattened HNLF (DF-HNLF), from which both data and pump spectra are carved out. The pump pulses for the FWM process are filtered at $1545 \mathrm{~nm}$ by using a 5-nm OBPF for the 320-Gbit/s case or a 13-nm OBPF for the 640-Gbit/s case. The $10-\mathrm{GHz}$ pulse train for the data signal is obtained by filtering the supercontinuum at $1557 \mathrm{~nm}$ with a 13-nm OBPF for both cases. The data pulses are OOK encoded with a $10-\mathrm{Gbit} / \mathrm{s} 2^{7}-1$ PRBS pattern in a Mach-Zehnder modulator (MZM), and then multiplexed up to 320 or $640 \mathrm{Gbits} / \mathrm{s}$ by using a passive fiber-based delay line multiplexer. Both data and pump signals are dispersed in different lengths of DCF in order to achieve the desired spectral compression at the idler. Subsequently, the dispersed data and chirped pump are amplified and injected into a polarisationmaintaining HNLF (PM-HNLF). The PM-HNLF is $100 \mathrm{~m}$ long with zero dispersion wavelength $\lambda_{0}$ at $1545 \mathrm{~nm}$, dispersion slope $0.025 \mathrm{ps} /\left(\mathrm{nm}^{2} \mathrm{~km}\right)$, and nonlinear coefficient $\gamma \approx 10(\mathrm{~W} \cdot \mathrm{km})^{-1}$. At the PM-HNLF output, a narrow 0.3-nm tuneable OBPF extracts the converted 10-Gbit/s tributaries centered at different wavelengths in the idler signal. The time delay $\Delta t$ (see Fig. 8) is used to change the position between the center of the pump pulse and the OTDM channels, hence shifting their position in the frequency domain. The filtered tributaries are sent into a 10Gbit/s preamplified receiver for BER evaluation. The receiver and the PRBS generator are synchronized to the same clock signal extracted from the ERGO-PGL.

\section{B. 320-Gbit/s Case}

First, the OTDM-to-WDM (serial-to-parallel) conversion scheme was applied to a 320-Gbit/s OTDM signal [15]. This was done in order to see the bell-shaped spectrum envelope of the idler resulting from Gaussian pump pulses, as previously seen in Section III. Before dispersion, both data and pump pulses were Gaussian like with 1.3 ps FWHM. The dispersive fiber used was $20 \mathrm{~m} \mathrm{DCF}$ for the data and $48 \mathrm{~m} \mathrm{DCF}$ for the pump. The pump pulses at the input of the PM-HNLF were, thus, broadened up to $17.5 \mathrm{ps}$ FWHM. The average powers at the input of the PM-HNLF were $20.3 \mathrm{dBm}$ for the pump and $2.6 \mathrm{dBm}$ for the
320-Gbit/s data. Fig. 9(a) shows the resulting spectrum at the output of the PM-HNLF. As can be seen, the tributaries are successfully mapped to different wavelengths with $\sim 1.1 \mathrm{~nm}$ spacing. Nine different 10-Gbit/s tributaries from 1528.6 to $1537.7 \mathrm{~nm}$ were extracted using a $0.3 \mathrm{~nm}$ OBPF (see Fig. 9(b), top), and the corresponding BER curves are shown in Fig. 9(b), bottom. The performance is error free for all channels, with a penalty of $<1.6 \mathrm{~dB}$ compared to the 10-Gbit/s back-to-back (B2B) reference, extracted at the MZM output with the $0.3 \mathrm{~nm}$ OBPF. Only the 1528.6-nm tributary exhibits a larger penalty of $3.0 \mathrm{~dB}$, attributed to a $10-\mathrm{dB}$ lower conversion efficiency compared to the central tributaries.

\section{640-Gbit/s Case}

The OTDM-to-WDM conversion scheme was successfully performed also on a 640-Gbit/s OTDM system. The average input powers into the PM-HNLF were $24 \mathrm{dBm}$ for the pump and $15.5 \mathrm{dBm}$ for the $640-\mathrm{Gbit} / \mathrm{s}$ data. Before being dispersed, the transform-limited FWHM were 600 fs for the 640-Gbit/s data signal and 490 fs for the 10-GHz pump. Fig. 10 shows the output spectrum of the PM-HNLF, where a tributary channel spacing of $100 \mathrm{GHz}$ is achieved. The idler signal contains 19 tributaries mapped to different wavelengths in the range 1520 $1535 \mathrm{~nm}$. In this case, the 640-Gbit/s OTDM data and 10-GHz pump were dispersed in 15 and 36 m DCF [see Fig. 11(a)]. As can be noticed, the simulated result shown in Fig. 4 is similar to the measured one in Fig. 11(a), where in both cases the resulting WDM channel spacing is $100 \mathrm{GHz}$. The only difference is the DCF lengths used to disperse the data and linearly chirp the pump. This is related to the different pieces of DCF fibers, with presumably different characteristics, used in the experiment. By increasing the DCF lengths, it is possible to increase the spectral compression rate as well. This was seen in Section III and it is confirmed in Fig. 11(b). In this case, data and pump were dispersed in 23 and $48 \mathrm{~m} \mathrm{DCF}$, resulting in $0.55-\mathrm{nm}(69 \mathrm{GHz})$ channel spacing of the tributaries.

By changing the time delay between pump and data, it is possible to adjust the idler shown in Fig. 11(b) to the wavelength grid for 100-GHz DWDM as specified in [11]. Smaller channel spacing is possible, e.g., for $50-\mathrm{GHz}$ systems, but in this case a narrower OBPF is required in order to select the desired tributary channel. The eight tributaries from 1527.8 to $1533.6 \mathrm{~nm}$ are extracted by tuning the $0.3 \mathrm{~nm}$ OBPF, whose transfer function is shown in Fig. 11(a). The resulting spectra are shown in Fig. 12 and the corresponding BER curves in Fig. 13. For the best tributary, the performance is error free with penalty $<2.5 \mathrm{~dB}$ compared to the 10-Gbit/s B2B reference at $1557 \mathrm{~nm}$. As can be seen, the tributaries centered at the longer wavelengths have a worse sensitivity. This is attributed to the closer presence of the pump signal spectrum. To verify the integrity of the entire 640-Gbit/s OTDM signal, each tributary is extracted by keeping the $0.3-\mathrm{nm}$ OBPF fixed at $1529.5 \mathrm{~nm}$ and by tuning the time delay $(\Delta t)$ to extract each tributary. All 64 tributaries have errorfree performance with a sensitivity variation of $3.2 \mathrm{~dB}$ as shown in Fig. 13 (top).

Finally, it can be noticed that due to the narrow channel spacing and optical window used, DWDM systems require 


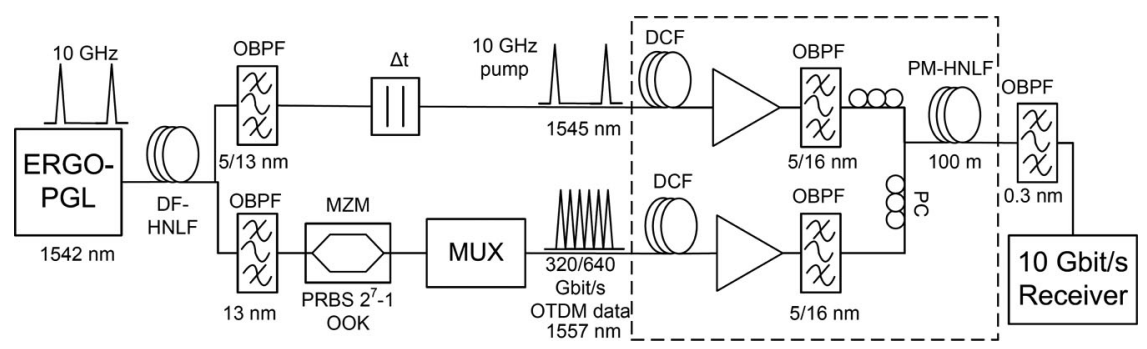

Fig. 8. Experimental setup for OTDM-to-WDM conversion for 320- and 640-Gbit/s OTDM systems. In the OBPF nomenclature, the "/" character conceptually divides the filter used for the 320- or 640-Gbit/s OTDM data setup.

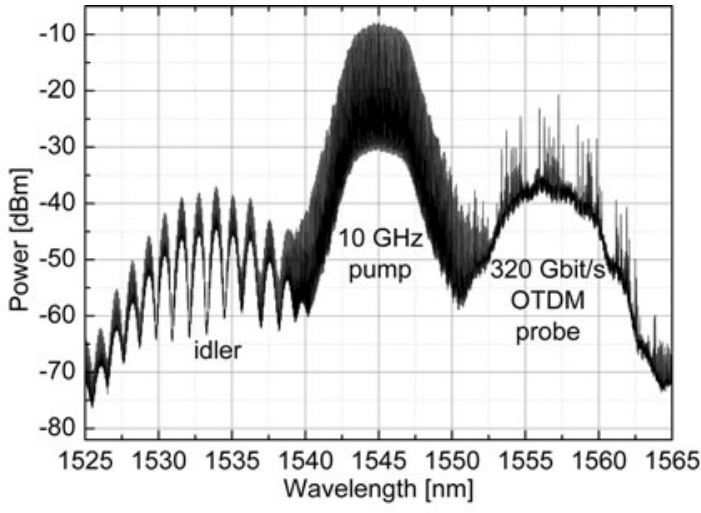

(a)

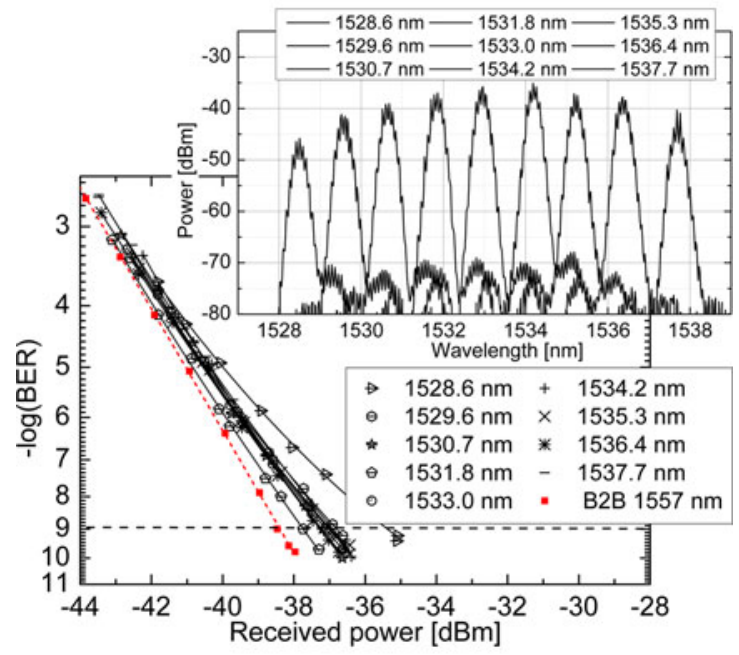

(b)

Fig. 9. System performance of 320-Gbit/s OTDM-to-WDM conversion. (a) Output spectrum of PM-HNLF. (b) top: 10-Gbit/s tributaries extracted at different wavelengths (resolution $0.01 \mathrm{~nm}$ ); bottom: BER curves of nine of the $10-\mathrm{Gbit} / \mathrm{s}$ tributaries from $1528.6 \mathrm{~nm}$ to $1537.7 \mathrm{~nm}$ and the reference back-toback (B2B) case.

well-controlled, cooled lasers to prohibit drift outside of a given DWDM optical channel. A reliable grid at $25 \mathrm{GHz}$ would require the network lasers to maintain accuracy over time and environment of at least $\pm 0.02 \mathrm{~nm}$ or even better. Such lasers are not easily available. But with the time-domain OFT technique implementation of serial-to-parallel conversion, what is required is the right compression, and to a certain extent the stability of the OTDM signal against time drifts. Once these requirements are achieved, then the right wavelength allocation at the

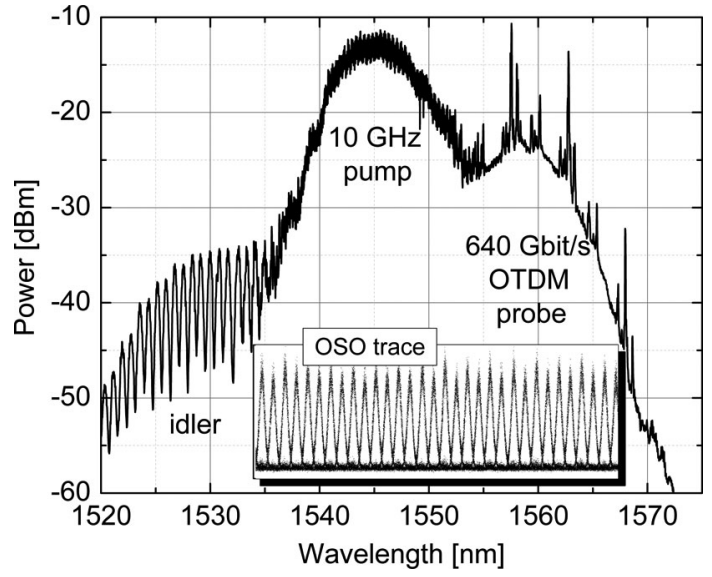

Fig. 10. Spectrum at the output of the PM-HNLF, for 100-GHz spacing of the idler channels. Inset: Optical sampling oscilloscope (OSO) trace of the $640-\mathrm{Gbit} / \mathrm{s}$ original data signal.

demultiplexer can be obtained by just changing the relative time delay between pump and OTDM signal, possibly controlled by a feedback mechanism.

\section{System Performance in Nonoptimum Data Dispersion}

In case the data accumulated dispersion and pump linear chirp are not optimized, then the condition $C=1 / D_{\text {acc }}$ does not hold any longer, resulting in a distorted time-to-frequency mapping. In order to investigate the sensitivity of the system in the case of nonoptimal conditions, the pump dispersion is kept at $36 \mathrm{~m}$ DCF and the data dispersion is changed by adding some extra lengths of single-mode fiber (SMF) or DCF to the optimal value of $15 \mathrm{~m}$ DCF. This was done by adding, respectively, 5, 10, or $12 \mathrm{~m} \mathrm{SMF}$, or 1 - or $2 \mathrm{~m} \mathrm{DCF}$. This would correspond to lowering the data dispersion by $4.7,9.5$, or $11.4 \%$ in the case of additional $\mathrm{SMF}^{3}$ or increasing it by 6.6 or $13.3 \%$ in the case of additional DCF. The deterioration of the system's performance can be seen in Fig. 14. Here are shown the BER curves of the extracted 10-Gbit/s tributaries using the $0.3 \mathrm{~nm}$ OBPF centered at $1529.5 \mathrm{~nm}$. As can be seen, the tolerance of the system to extra dispersion is in the order of $\pm 1 \mathrm{~m} \mathrm{DCF}(\sim \pm 7 \%)$. As the dispersion imbalance increases, the BER curve starts having an error floor (10 $\mathrm{m}$ SMF trace) resulting from intersymbol interference between neighboring channels.

\footnotetext{
${ }^{3} 7 \mathrm{~m} \mathrm{SMF}$ are supposed to compensate for $1 \mathrm{~m} \mathrm{DCF}$.
} 


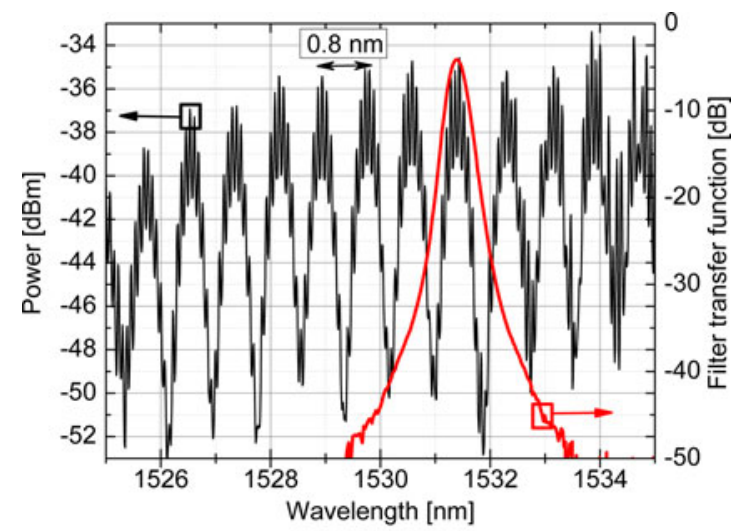

(a)

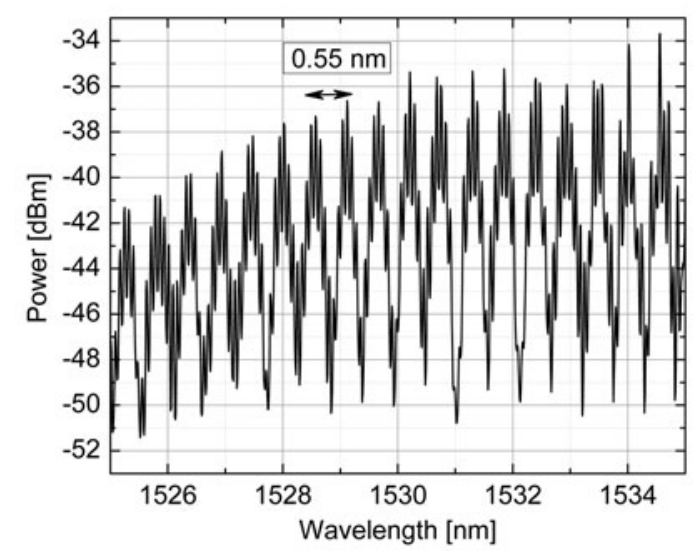

(b)

Fig. 11. Idler spectra (at the output of the PM-HNLF) when the 640-Gbit/s OTDM data and the 10-GHz pump are dispersed, respectively, in (a) 15 and $36 \mathrm{~m} \mathrm{DCF}$, and (b) 23 and $48 \mathrm{~m}$ DCF (resolution $0.05 \mathrm{~nm}$ ). (a) Transfer function of the $0.3 \mathrm{~nm}$ OBPF.

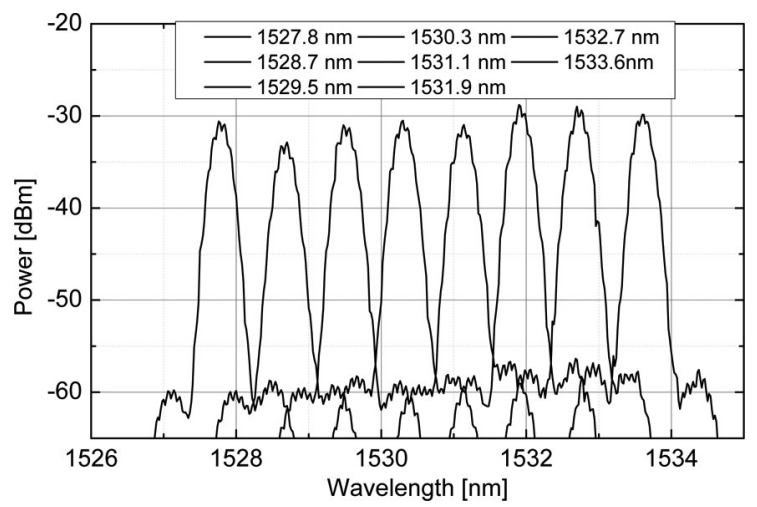

Fig. 12. Spectra of the 10-Gbit/s tributaries extracted at different wavelengths by using the 0.3-nm OBPF, whose transfer function is shown in Fig. 11(a).

\section{CONCLUSION}

This paper has reported on the utilization of the time-domain OFT for serial (OTDM) to parallel (WDM) conversion. The technique is based on time-to-frequency mapping implemented by an FWM process between the dispersed OTDM data and linearly chirped pump pulses. This technique was successfully demonstrated for a 640-Gbit/s OTDM signal, where eight tributaries were simultaneously converted to $0.8-\mathrm{nm}(100 \mathrm{GHz})-$

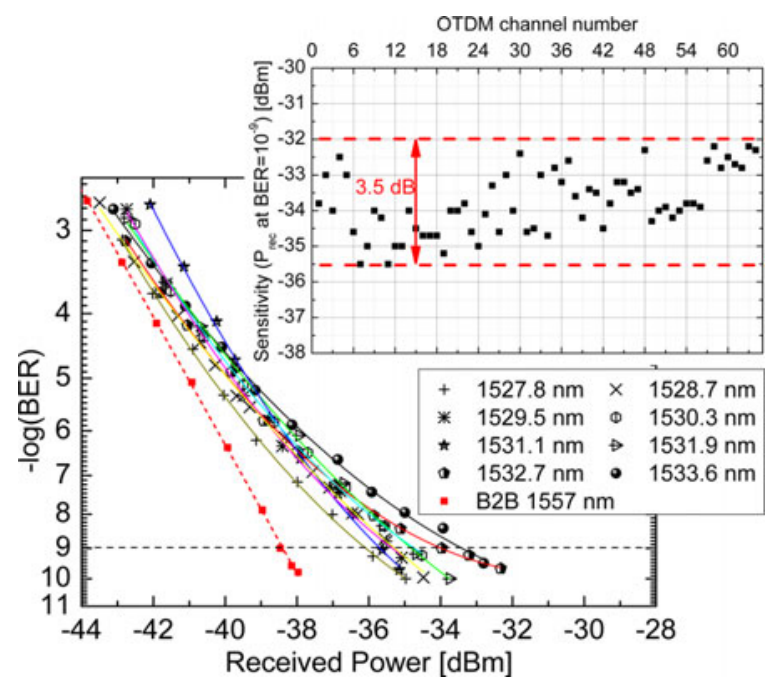

Fig. 13. Bottom: BER measurement for the 10-Gbit/s tributaries extracted at different wavelengths. Top: sensitivity of all the 64 OTDM channels, measured by scanning the OTDM signal in the time domain and keeping the $0.3-\mathrm{nm}$ OBPF fixed at $1529.5 \mathrm{~nm}$.

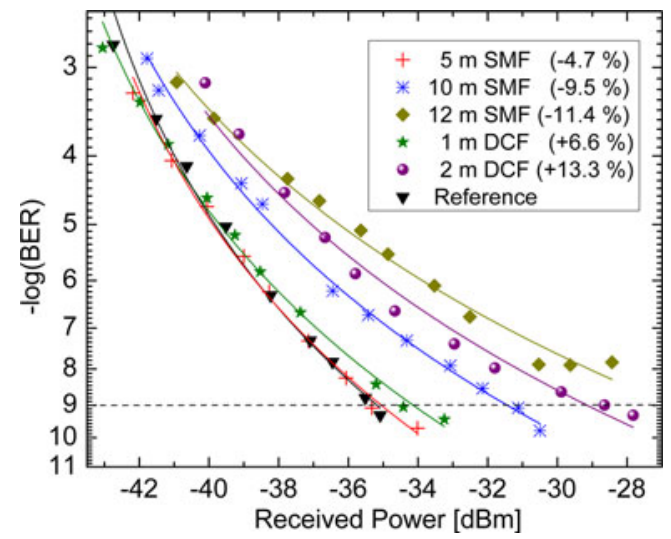

Fig. 14. BER in case the 640-Gbit/s signal dispersion is changed from the optimal (reference curve) value of $15 \mathrm{~m}$ DCF. The deviation is expressed both in terms of dispersion percentage variation and amount and type of fiber added. The pump dispersion was kept at $36 \mathrm{~m}$ DCF.

spaced 10-Gbit/s WDM channels showing error-free performance with low penalty. The process should be independent of tributary data modulation and channel spacing can be controlled by manipulating the amount of data dispersion and pump linear chirp. Numerical simulations show scalability to even higher bit rates, and indicate the possibility of converting up to half of all OTDM tributaries into well-equalized WDM channels by using linearly chirped flat-top pump pulses. This OTDM-to-WDM conversion technique can enable a significant reduction in the complexity of an OTDM receiver.

\section{ACKNOWLEDGMENT}

The authors would like to thank OFS Fitel Denmark ApS for kindly providing the DF-HNLF and PM-HNLF.

\section{REFERENCES}

[1] H. C. H. Mulvad, L. K. Oxenløwe, M. Galili, A. T. Clausen, L. GrünerNielsen, and P. Jeppesen, "1.28 Tbit/s single-polarisation serial OOK optical data generation and demultiplexing," Electron. Lett., vol. 45, no. 5, pp. 280-281, Feb. 2009. 
[2] K. Uchiyama, H. Takara, K. Mori, and T. Morioka, "160 Gbit/s all-optical time-division demultiplexing utilising modified multiple-output OTDM demultiplexer (MOXIC)," Electron. Lett., vol. 38, no. 20, pp. 1190-1191, Sep. 2002.

[3] C. S. Brès, A. O. J. Wiberg, B. P. Kuo, J. M. Chavez-Boggio, C. F. Marki, N. Alic, and S. Radic, "Single-gate 320 -to- $8 \times 40 \mathrm{~Gb} / \mathrm{s}$ demultiplexing," presented at the Optical Fiber Communication, San Diego, CA, 2009, Postdeadline Paper PDPA4.

[4] S. Liu, K. J. Lee, F. Parmigiani, M. Ibsen, P. Petropoulos, D. Richardson, and K. Gallo, "OTDM to WDM format conversion based on cascaded SHG/DFG in a single PPLN waveguide," presented at the Optical Fiber Communication, San Diego, CA, 2010, Paper OWF4.

[5] C. V. Bennett and B. H. Kolner, "Principles of parametric temporal imaging-Part I: System configurations," IEEE J. Quantum Electron., vol. 36, no. 4, pp. 430-437, Apr. 2000.

[6] M. T. Kauffman, W. C. Banyal, A. A. Godil, and D. M. Bloom, "Timeto-frequency converter for measuring picosecond optical pulses," Appl. Phys. Lett., vol. 64, no. 3, pp. 270-272, Jan. 1994.

[7] B. H. Kolner, "Space-time duality and the theory of temporal imaging," IEEE J. Quantum Electron., vol. 30, no. 8, pp. 1951-1963, Aug. 1994.

[8] J. W. Goodman, Introduction to Fourier Optics, 2nd ed. ed. New York: McGraw-Hill, 1996.

[9] K. N. Berger, B. Levit, S. Atkins, and B. Fischer, "Time-lens-based spectral analysis of optical pulses by electrooptic phase modulation," Electron Lett., vol. 30, no. 36, pp. 1644-1646, Sep. 2000.

[10] T. Hirooka and M. Nakazawa, "Optical adaptive equalization of highspeed signals using time-domain optical Fourier transformation," $J$ Lightw. Technol., vol. 24, no. 7, pp. 2530-2540, Jul. 2006.

[11] Spectral Grids for WDM Applications: DWDM Frequency Grid, ITU-T G.694.1 May 2002.

[12] G. Bosco, A. Carena, V. Curri, R. Gaudino, P. Poggiolini, and S. Benedetto, "Suppression of spurious tones induced by the split-step method in fiber systems simulation," IEEE Photon. Technol. Lett., vol. 12, no. 5, pp. 489 491, May 2000.

[13] E. Palushani, L. K. Oxenløwe, M. Galili, H. C. H. Mulvad, and A. T.Clausen, P. Jeppesen, "Flat-top pulse generation by the optical Fourier transform technique for ultra-high-speed signal processing," IEEE $J$. Quantum Electron., vol. 45, no. 11, pp. 1317-1324, Nov. 2009.

[14] L. G. Nielsen, M. Wandel, P. Kristensen, C. Jørgensen, L.V. Jørgensen, B. Edvold, B. Pálsdóttir, and D. Jakobsen, "Dispersion-Compensating Fibers," J. Lightw. Technol., vol. 23, no. 11, pp. 3566-3579, Nov. 2005.

[15] H. C. H. Mulvad, E. Palushani, M. Galili, J. Xu, H. Hu, A. Clausen, L. Oxenløwe, and P. Jeppesen, "OTDM-WDM conversion based on timedomain optical Fourier transformation with spectral compression," presented at the Optical Fiber Communication, 2011, Paper OThN2.

Evarist Palushani was born in Shkoder, Albania, in 1983. He received the B.Sc. degree in computer engineering from the University of Padua, Padua, Italy, in 2005 and the M.Sc. degree in telecommunications from the Technical University of Denmark (DTU), Kongens Lyngby, Denmark, in 2008. He is currently working toward the Ph.D. degree in the NOSFERATU project in the High-Speed Optical Communications Group at DTU Fotonik, Department of Photonics Engineering, DTU, where he is involved in research on high-speed signal processing and Terabit/s optical communication systems.

Hans Christian Hansen Mulvad was born in Copenhagen, Denmark, in 1976. He received the M.Sc. degree in physics from the University of Copenhagen, Copenhagen, Denmark, in 2004, and the Ph.D. degree from DTU Fotonik, Department of Photonics Engineering, Technical University of Denmark, Kongens Lyngby, Denmark, in 2008 .

$\mathrm{He}$ is currently an Assistant Professor in the High-Speed Optical Communications Group at DTU Fotonik, where he is involved in research on high-speed signal processing and Terabit/s optical communication systems.

Michael Galili was born in Aabenraa, Denmark in 1977. He received the M.Sc. degree in engineering in applied physics with main focus on optics and semiconductor physics from the Technical University of Denmark, Kongens Lyngby, Denmark, in 2003, and the Ph.D. degree in optical communications and signal processing from the COM department of the Technical University of Denmark, Kongens Lyngby, Denmark in 2007, now DTU Fotonik. The main focus of the Ph.D. work was on optical signal processing of high-speed optical data signals.

$\mathrm{He}$ is currently with the Technical University of Denmark as a Postdoc Fellow. He is the first author or coauthor of more than 30 peer-reviewed scientific publications.
Hao Hu received the Ph.D. degree in optical communications from Tianjin University, Tianjin, China in 2009.

From 2007 to 2008, he was a Visiting Scientist at Fraunhofer Institute for Telecommunications, Heinrich-Hertz-Institute, Berlin, Germany. He is currently a Postdoctoral Researcher at DTU Fotonik, Department of Photonics Engineering, Technical University of Denmark, Kongens Lyngby, Denmark. His current research interests include Terabit Ethernet and ultrahigh-speed optical signal processing and transmission on $640 \mathrm{Gbits} / \mathrm{s}$ and beyond. He has authored or coauthored more than 60 peer-reviewed publications.

Leif Katsuo Oxenløwe received the B.Sc. degree in physics and astronomy from Niels Bohr Institute, University of Copenhagen, Copenhagen, Denmark, in 1996, the International Diploma of Imperial College of Science, Technology, and Medicine, London, U.K., in 1998, the M.Sc. degree from the University of Copenhagen, Copenhagen, Denmark, in 1998, and the Ph.D. degree from the Technical University of Denmark, Kongens Lyngby, Denmark, in 2002.

$\mathrm{He}$ is currently an Associate Professor at DTU Fotonik, Department of Photonics Engineering, Technical University of Denmark, where he is the group leader of the High-Speed Optical Communications Group. He is involved in experimental research in the field of ultrafast optical communications (above $160 \mathrm{Gbits} / \mathrm{s})$. He has been involved within the EU IST project TOPRATE, and the Danish research council financed projects SCOOP and ULTRA-NET. From August 2007, he was with the project NANO-COM, and from January 2009, he was with the project NOSFERATU. The latter two projects deal with nanotechnology-based solutions for ultrahigh bit rate communications and Terabit/s Ethernet solutions. He has authored or coauthored more than 120 peerreviewed publications.

Anders T. Clausen received the M.Sc. degree in electrical engineering from the former Electromagnetics Institute (EMI), Technical University of Denmark (DTU), Lyngby, in 1997 and the Ph.D. degree from DTU Fotonik, DTU, in 2007.

He is an Associate Professor at DTU Fotonik. He was born in Copenhagen, Denmark, in 1967. He joined EMI as a Research Associate in 1997, working on optical signal processing. He has participated in a number of European projects, such as HIGHWAY, REPEAT and METEOR and other national projects. He was the head of the Optical Time Division Multiplexing (OTDM) group from 2001 to 2008. His doctoral work at COM focused on experimental and theoretical investigations of systems with potential for terabit capacity. He is the first author or coauthor of more than 100 peer-reviewed scientific publications. His research interests are primarily focused on ultrahigh-speed signal processing at bit rates of $640 \mathrm{Gbit} / \mathrm{s}$ and beyond.

Palle Jeppesen (M'69) was born in 1941 in Vordingborg, Denmark. He received the M.Sc. degree in electrophysics in 1967 the Lic. Tech. (Ph.D.) degree in 1970, and the Dr. Tech. (D.Sc.) degree in 1978 all the Technical University of Denmark (DTU), Kongens Lyngby, Denmark.

From 1968 to 1969, he was a Research Associate at Cornell University, Ithaca, NY, and from 1969 to 1970, a Project Engineer at Cayuga Associates, Ithaca, NY; at both places he did research in the field of GaAs Gunn effect microwave oscillators. From 1970 to 1998, he was an Assistant, Associate, Research and Full Professor at the Department of Electromagnetic Systems (EMI), Technical University of Denmark, first in microwave electronics, and since 1974, in optical communications. From 1974 to 1988 at EMI, he was the Head of Optogroup, and from 1988 to 1998, he was the Head of the Center for Broadband Telecommunications. From 1982 to 1984, he was also a Part-time Manager of R\&D at NKT Elektronik, now Draka Denmark Optical Cable, OFS Fitel Denmark and Tellabs Denmark. Since 1998, he has been a Professor of Optical Communications at Research Center COM (now DTU Fotonik), where he is currently heading the Optical Transmission and Network Elements Group and acting as a Coordinator of the five groups in the Communications Technology Research Cluster, DTU Fotonik. His current research interests include optical multilevel modulation formats and 160-640-Gbit/s optical communication. He is currently a Supervisor for six Ph.D. students.

Dr. Jeppesen has received the P. Gorm Petersens Memorial Stipend in 1974 the Esso Prize in 1978, the Villum Kann Rasmussen Prize in 1988, and the Alexander Foss Gold Medal in 2005. He has been a member of numerous evaluation committees at Technical University of Denmark and in other countriesin 2002 for Styrelsen för Strategisk Forskning, in 2003 and 2004 for Vetenskapsrådet and for Chalmers Tekniska Högskolan in Sweden, respectively, and in 2007 the peer review panel for European Research Council Starting Grants and again for Styrelsen för Strategisk Forskning in Sweden. 